\title{
Existencia de soluciones débiles para una clase de sistemas elípticos semilineales
}

\section{Marlon Yvan Tineo Condeña ${ }^{1}$}

Resumen: Este artículo resume las contribuciones principales de la tesis con el título "Existencia de soluciones para una clase de sistemas elípticos semilineales". Esta tesis se centra en una exposición didáctica del artículo publicado por Afrouzi, G., Mirzapour, A. and Zographopoulos, N. [1], cuyo objetivo es probar la existencia de soluciones débiles para una clase de sistemas elípticos semilineales potenciales de la forma

$$
\left\{\begin{array}{rr}
-\operatorname{div}(a(x) \nabla u)=\lambda F_{u}(x, u, v) & \text { en } \Omega \\
-\operatorname{div}(b(x) \nabla v)=\lambda F_{v}(x, u, v) & \text { en } \Omega \\
u=v=0 & \text { sobre } \partial \Omega
\end{array}\right.
$$

donde el dominio $\Omega$ es un dominio acotado en $\mathbb{R}^{N}(N>2)$, de frontera bien regular, los pesos $a(x), b(x)$ son pesos medibles no negativas sobre $\Omega,\left(F_{u}, F_{v}\right)=\nabla F$ representa el gradiente de $F$ en las variables $(u, v) \in \mathbb{R}^{2}$ y $\lambda$ es un parámetro positivo. Palabras clave: ecuación elíptica degenerada; sistema elíptico semilineal; teorema del paso de la montaña.

\section{Existence of weak solutions for a class of systems semilinear elliptics}

Abstract: This article summarizes the main contributions of the thesis with the title "Existence of solutions for a class of semilinear elliptical systems ". This thesis focuses on a didactic exhibition of the article published by Afrouzi, G., Mirzapour, A. and Zographopoulos, N.[1], whose objective is to prove the existence of weak solutions to a class of semilinear potential elliptic systems of the form

$$
\left\{\begin{array}{rr}
-\operatorname{div}(a(x) \nabla u)=\lambda F_{u}(x, u, v) & \text { in } \Omega \\
-\operatorname{div}(b(x) \nabla v)=\lambda F_{v}(x, u, v) & \text { in } \Omega \\
\mathrm{u}=\mathrm{v}=0 & \text { on } \partial \Omega
\end{array}\right.
$$

where the domain $\Omega$ is a bounded domain in $\mathbb{R}^{N}(N>2)$, regular border, the weights $a(x), b(x)$ are measurable nonnegative weights on $\Omega,\left(F_{u}, F_{v}\right)=\nabla F$ stands for the gradient of $F$ in the variables $(u, v) \in \mathbb{R}^{2}$ and $\lambda$ is a positive parameter.

Keywords: degenerate elliptic equations, semilinear potential elliptic system, Mountain Pass Theorem.

Recibido: 06/03/2018. Aceptado: 18/05/2018. Publicado online: 30/06/2018.

(CLos autores. Este artículo es publicado por la Revista PESQUIMAT de la Facultad de Ciencias Matemáticas, Universidad Nacional Mayor de San Marcos. Este es un artículo de acceso abierto, distribuido bajo los términos de la licencia Creative Commons Atribucion-No Comercia-Compartir Igual 4.0 Internacional.(http://creativecommons.org/licenses/by-nc-sa/4.0/) que permite el uso no comercial, distribución y reproducción en cualquier medio, siempre que la obra original sea debidamente citada. Para información, por favor póngase en contacto con revistapesquimat.matematica@unmsm.edu.pe

${ }^{1}$ UNMSM, Facultad de Ciencias Matemáticas. e-mail: p140277@unmsm.edu.pe 


\section{Introducción}

En el presente artículo resumo mi trabajo de tesis presentado a consideración del cuerpo docente de la Facultad de Ciencias Matemáticas de la Universidad Nacional Mayor de San Marcos, como parte de los requisitos para obtener el Grado Académico de Magíster en Matemática Pura, donde se da una explicación didáctica y una aplicación de los resultados obtenidos en el artículo publicado por G.A. Afrouzi, M. Mirzapour y N.B. Zographopoulos [1], el cual consiste en probar la existencia de soluciones para una clase de sistemas elípticos semilineales potenciales de la forma:

$$
\left\{\begin{array}{rr}
-\operatorname{div}(a(x) \nabla u)=\lambda F_{u}(x, u, v) & \text { en } \Omega \\
-\operatorname{div}(b(x) \nabla v)=\lambda F_{v}(x, u, v) & \text { en } \Omega \\
\mathrm{u}=\mathrm{v}=0 & \text { en } \partial \Omega
\end{array}\right.
$$

donde $\Omega$ es un dominio acotado de $\mathbb{R}^{N}$, de frontera bien regular, $N>2$, los pesos $a(x), b(x)$ son funciones medibles no negativos sobre $\Omega,\left(F_{u}, F_{v}\right)=\nabla F$ representa el gradiente de $F$ en las variables $(u, v) \in \mathbb{R}^{2}$ y $\lambda$ es un parámetro positivo.

Esta clase de sistemas modela varios fenómenos físicos relacionados con el equilibrio de medios continuos, por ello muchos autores han estudiado la existencia de soluciones débiles no triviales para tales problemas elípticos, en [3], [4], [5], [6], [9], [12], [?], [14] y en [7, pp. 79] por Dautray y Lions.

Para probar la existencia de soluciones se necesita algunas condiciones sobre los pesos $a(x), b(x)$ y el funcional $F(x, u, v)$. Los pesos $a, b \in L_{L o c}^{1}(\Omega)$, satisfacen

$$
a^{-s}, b^{-s} \in L^{1}(\Omega), s \in\left\langle\frac{N}{2}, \infty\right\rangle \bigcap[1, \infty\rangle .
$$

Con $s$ definimos

$$
2_{s}=\frac{2 s}{s+1}, 2_{s}^{*}=\frac{N 2_{s}}{N-2_{s}}=\frac{N 2 s}{N(s+1)-2 s}>2,
$$

tal como se mostrará en la Observación 2.4 para $\mathrm{p}=2$.

Suponemos que $\mathrm{F}$ es un $C^{1}$ - Funcional sobre $\Omega \times \mathbb{R} \times \mathbb{R} \longrightarrow \mathbb{R}$ y satisfaciendo las siguientes condiciones:

(F1) Existen constantes positivas $c_{1}, c_{2}$ tal que :

$$
\begin{aligned}
& \left|F_{t}(x, t, s)\right| \leq c_{1}|t|^{\gamma}|s|^{\delta+1} \\
& \left|F_{s}(x, t, s)\right| \leq c_{2}|t|^{\gamma+1}|s|^{\delta}
\end{aligned}
$$

para todo $(t, s) \in \mathbb{R}^{2}$, casi todo punto $x \in \Omega$ y algunos $\gamma, \delta>1$ tales que $\frac{\gamma+1}{p}+\frac{\delta+1}{q}=1 \mathrm{y}$ $\gamma+1<p<2_{s}^{*}, \delta+1<q<2_{s}^{*}$.

(F2) Existen constantes positivas $c$ y $2<\alpha, \beta<2_{s}^{*}$ tal que

$$
|F(x, t, s)| \leq c\left(1+|t|^{\alpha}+|s|^{\beta}\right)
$$

para todo $x \in \Omega$ y $t, s \in \mathbb{R}$.

(F3) Existen $R>0, \theta$ y $\theta^{\prime} \operatorname{con} \frac{1}{2_{s}^{*}}<\theta, \theta^{\prime}<\frac{1}{2}$ tal que

$$
0<F(x, t, s) \leq \theta t F_{t}(x, t, s)+\theta^{\prime} s F_{s}(x, t, s)
$$

para todo $x \in \bar{\Omega} \mathrm{y}|t|,|s| \geq R$

(F4) Existe $\bar{\alpha}>2, \bar{\beta}>2$ y $\epsilon>0$ tal que

$$
|F(x, t, s)| \leq c\left(|t|^{\bar{\alpha}}+|s|^{\bar{\beta}}\right)
$$

para todo $x \in \bar{\Omega} \mathrm{y}|t|,|s| \leq \epsilon$ 


\section{Preliminares}

Definición 2.1 Sea $1<p<\infty$. Definimos la clase de funciones de peso $B_{p}(\Omega)$ a las funciones de peso $\omega$ que satisface la condición:

$$
w^{\frac{-1}{p-1}} \in L_{l o c}^{1}(\Omega)
$$

Definición 2.2 Sea $\omega$ una función peso. Para $1 \leq p<\infty$ definimos $L^{p}(\Omega, \omega)$ como el espacio de funciones medibles u definidas sobre $\Omega$ tales que

$$
\|u\|_{L^{p}(\Omega, \omega)}:=\left(\int_{\Omega}|u(x)|^{p} \omega(x) d x\right)^{1 / p}<\infty .
$$

Así, $L^{p}(\Omega, \omega)$ es un espacio de Banach con la norma dada por (3).

Observación 2.1 Sea $1<p<\infty$ y $\omega \in B_{p}(\Omega)$. Entonces

$$
L^{p}(\Omega, \omega) \subset L_{L o c}^{1}(\Omega)
$$

Además, identificando una distribución regular de $D^{\prime}(\Omega)$ con una función de $L_{L o c}^{1}(\Omega)$ obtenemos

$$
L^{p}(\Omega, \omega) \subset L_{L o c}^{1}(\Omega) \subset D^{\prime}(\Omega) \text { para } 1<p .
$$

Definición 2.3 Sean $1 \leq p<\infty$. Si $\omega=\left\{\omega_{o}, \omega_{1}, \ldots, \omega_{N}\right\}$ es una familia de pesos en $B_{p}(\Omega)$ entonces se define el espacio de Sobolev con peso

$$
W^{1, p}(\Omega, \omega)=\left\{u \in L^{p}\left(\Omega ; \omega_{o}\right) / \frac{\partial u}{\partial x_{i}} \in L^{p}\left(\Omega ; \omega_{i}\right), \forall i=1, \ldots, N\right\} .
$$

Este espacio es de Banach con la siguiente norma

$$
\|u\|_{W^{1, p}(\Omega, \omega)}=\left(\int_{\Omega}|u(x)|^{p} \omega_{o}(x) d x+\sum_{i=1}^{N} \int_{\Omega}\left|\frac{\partial u}{\partial x_{i}}(x)\right|^{p} \omega_{i}(x) d x\right)^{1 / p} .
$$

Observación 2.2 Para nuestros propósitos, necesitamos de los espacios $W_{o}^{1, p}(\Omega ; a)$ definidos como la clausura de $C_{o}^{\infty}(\Omega)$ con respecto a la norma (7). Para esto, es necesaria la inclusión

$$
C_{o}^{\infty}(\Omega) \subset W^{1, p}(\Omega ; \omega)
$$

el cual es satisfecha si

$$
\omega_{i} \in L_{L o c}^{1}(\Omega) \text { para todo } i=0, \ldots, N
$$

Definición 2.4 Sean $1<p<\infty$ y la familia de funciones de peso $\omega$ tal que $\omega_{i} \in B_{p}(\Omega)$ para $i=1, \ldots, N$ y $\omega_{i} \in L_{\text {Loc }}^{1}(\Omega)$ para todo $i=0, \ldots, N$. Entonces definimos

$$
W_{o}^{1, p}(\Omega ; \omega)=\overline{C_{o}^{\infty}(\Omega)},
$$

donde la clausura es tomada con respecto a la norma (7). 
Observación 2.3 Para la familia de pesos:

$$
\omega_{o}=1, \omega_{1}(x)=\omega_{2}(x)=\ldots=\omega_{N}=a(x),
$$

el espacio de sobolev con peso $W^{1, p}(\Omega ; a)$ es normado por:

$$
\|u\|_{W^{1, p}(\Omega, a)}=\left(\int_{\Omega}|u(x)|^{p} d x+\int_{\Omega}|\nabla u(x)|^{p} a(x) d x\right)^{1 / p} .
$$

Supongamos que la función de peso $a(x)$ satisface

$$
\begin{gathered}
a^{\frac{-1}{p-1}} \in L_{L o c}^{1}(\Omega), \\
a \in L_{L o c}^{1}(\Omega),
\end{gathered}
$$

también la condición

$$
a^{-s} \in L^{1}(\Omega)
$$

con un cierto $s>0$ el cual será especificado despues. Introducimos el parámetro

$$
p_{s}=\frac{p s}{s+1}<p .
$$

Afirmación $1: W^{1, p}(\Omega ; a) \hookrightarrow W^{1, p_{s}}(\Omega)$.

En efecto, usando la desigualdad de Hölder con el parámetro $r=\frac{s+1}{s}=\frac{p}{p_{s}}$ obtenemos

$$
\begin{aligned}
\int_{\Omega}\left|\frac{\partial u}{\partial x_{i}}\right|^{p_{s}} d x & =\int_{\Omega}\left|\frac{\partial u}{\partial x_{i}}\right|^{p_{s}} a^{\frac{p_{s}}{p}} a^{\frac{-p_{s}}{p}} d x \\
& \leq\left(\int_{\Omega}\left|\frac{\partial u}{\partial x_{i}}\right|^{p} a d x\right)^{\frac{p_{s}}{p}}\left(\int_{\Omega} a^{-s} d x\right)^{\frac{1}{s+1}}=c^{\prime}\left\|\frac{\partial u}{\partial x_{i}}\right\|_{L^{p}(\Omega ; a)}^{p_{s}}
\end{aligned}
$$

Y si $\Omega$ es un dominio acotado conseguimos: $u \in W^{1, p}(\Omega ; a)$ implica $u \in W^{1, p_{s}}(\Omega)$, esto es,

$$
\|u\|_{W^{1, p_{s}(\Omega)}} \leq c^{\prime}\|u\|_{W^{1, p}(\Omega ; a)} \text { para } p_{s}<p .
$$

Afirmación $2: W^{1, p}(\Omega ; a) \hookrightarrow L^{r}(\Omega)$ donde $1 \leq r \leq p_{s}^{*}=\frac{N p_{s}}{N-p_{s}}$ para $p s<N(s+1)$.

En efecto, usando el resultado de la afirmación $1 W^{1, p}(\Omega ; a) \hookrightarrow W^{1, p_{s}}(\Omega)$ y la inmersión de Sobolev usual $W^{1, p_{s}}(\Omega) \hookrightarrow L^{r}(\Omega)$ obtenemos el resultado.

Afirmación 3 : La inmersión $W^{1, p}(\Omega ; a) \stackrel{c}{\hookrightarrow} L^{r}(\Omega)$ es compacta para $1 \leq r<p_{s}^{*}$.

En efecto, usando el resultado de la Afirmación $1 W^{1, p}(\Omega ; a) \hookrightarrow W^{1, p_{s}}(\Omega)$ y la inmersión compacta de Sobolev usual $W^{1, p_{s}}(\Omega) \stackrel{c}{\hookrightarrow} L^{r}(\Omega)$ obtenemos el resultado.

Observación 2.4 Si $s>\frac{N}{p}$ entonces $p_{s}^{*}>p$ y consecuentemente por la afirmación 3

$$
W^{1, p}(\Omega ; a) \stackrel{c}{\hookrightarrow} L^{p}(\Omega) \text { para } s>\frac{N}{p} .
$$

Asumimos que (15) vale si

$$
a^{-s} \in L^{1}(\Omega) \text { con } s \in\left(\frac{N}{p}, \infty\right) \cap\left[\frac{1}{p-1}, \infty\right)
$$


ya que, de acuerdo con la definición 2.3, debemos suponer también que $s \geq \frac{1}{p-1}$ debido a que $a^{\frac{-1}{p-1}} \in L_{l o c}^{1}(\Omega)$. Consideremos el espacio $W^{1, p}(\Omega ; a)$ y su subespacio $W_{o}^{1, p}(\Omega ; a)$ con $\Omega$ un dominio acotado entonces las inmersiones derivadas para $W^{1, p}(\Omega ; a)$, también valen para $W_{o}^{1, p}(\Omega ; a)$.

Para $p_{s}^{*}>p$ tenemos, en virtud de la inmersión de Sobolev usual(Teorema Sobolev-GagliardoNiremberg) $W_{o}^{1, p_{s}}(\Omega) \hookrightarrow L^{p_{s}^{*}}$, que

$$
\begin{aligned}
\left(\int_{\Omega}|u(x)|^{p} d x\right)^{\frac{1}{p}} & \leq c_{1}\left(\int_{\Omega}|u(x)|^{p_{s}^{*}} d x\right)^{\frac{1}{p_{s}^{*}}} \\
& \leq c_{2}\left(\int_{\Omega}\left(|u(x)|^{p_{s}}+|\nabla u(x)|^{p_{s}}\right) d x\right)^{\frac{1}{p_{s}}} .
\end{aligned}
$$

La siguiente desigualdad de Friedrichs en $W_{o}^{1, p}(\Omega)$

$$
\int_{\Omega}\|u\|^{q} d x \leq c \int_{\Omega}\|\nabla u\|^{q} d x
$$

nos proporciona para $q=p_{s}$

$$
\left(\int_{\Omega}\left(|u(x)|^{p_{s}}+|\nabla u(x)|^{p_{s}}\right) d x\right)^{\frac{1}{p_{s}}} \leq c_{3}\left(\int_{\Omega}|\nabla u(x)|^{p_{s}} d x\right)^{\frac{1}{p_{s}}},
$$

procediendo como en la afirmación 1, mostramos que

$$
\begin{aligned}
\left(\int_{\Omega}|\nabla u(x)|^{p_{s}} d x\right)^{\frac{1}{p_{s}}} & \leq\left[\int_{\Omega}|\nabla u(x)|^{p_{s}} a^{\frac{p_{s}}{p}} a^{\frac{-p_{s}}{p}} d x\right]^{\frac{1}{p_{s}}} \\
& \leq\left[\left(\int_{\Omega}|\nabla u(x)|^{p} a(x) d x\right)^{\frac{p_{s}}{p}}\left(\int_{\Omega} a^{-s}(x) d x\right)^{\frac{1}{s+1}}\right]^{\frac{1}{p_{s}}} \\
& =\left(\int_{\Omega}|\nabla u(x)|^{p} a(x) d x\right)^{\frac{1}{p}}\left(\int_{\Omega} a^{-s}(x) d x\right)^{\frac{1}{p \cdot s}}
\end{aligned}
$$

inmediatamente conseguimos la desigualdad de Friedrichs con peso

$$
\int_{\Omega}|u(x)|^{p} d x \leq c_{4} \int_{\Omega}|\nabla u(x)|^{p} a(x) d x .
$$

Finalmente obtenemos la expresión

$$
\|u\|_{W_{o}^{1, p}(\Omega ; a)}=\left(\int_{\Omega}|\nabla u(x)|^{p} a(x) d x\right)^{1 / p}
$$

que es una norma sobre el espacio $W_{o}^{1, p}(\Omega ; a)$ equivalente con la norma (11).

Teorema 2.1 (Principio del mínimo) Sea E un espacio de Hilbert (o mas general, considere un espacio de Banach reflexivo) y supongamos que un funcional $J: E \longmapsto \mathbb{R}$, verifica:

(M1) J es débilmente semicontinuo inferiormente

(M2) $J$ es coercivo (esto es $J(u) \rightarrow+\infty$ cuando $\|u\| \rightarrow \infty$ ),

entonces

Jes acotado inferiormente y existe $u_{o} \in E$ tal que $J\left(u_{o}\right)=\inf _{x \in E} J(x)$. 
Teorema 2.2 (Teorema del paso de la montaña) Sea $E$ un espacio de Banach Real y sea $J \in$ $C^{1}(E, \mathbb{R})$, un operador que satisface la condición de Palais-Smale. Suponga que $J(0)=0$ y que se satisfacen

(PM1) existen constantes positivas $\rho$ y $\sigma$ tales que $\left.J\right|_{\partial B_{\rho}(0)} \geq \sigma$

(PM2) existe un $w \in E-\overline{B_{\rho}(0)}$ tal que $J(w) \leq 0$,

entonces $J$ posee un valor crítico $c \geq \sigma$. Además c puede ser caracterizado como.

$$
c=\inf _{g \in \Gamma} \operatorname{máx}_{u \in g([0,1])} J(u)
$$

donde $\Gamma=\{g \in C([0,1], E) / g(0)=0, g(1)=w\}$

\section{Resultados de existencia}

Proposición 3.1 Dados los pesos a, b, definidos como en (16), entonces los funcionales

$$
\|u\|_{a}^{2}=\int_{\Omega} a(x)|\nabla u|^{2} d x y\|v\|_{b}^{2}=\int_{\Omega} b(x)|\nabla v|^{2} d x, \forall u, v \in C_{o}^{\infty},
$$

son normas que provienen de un producto interno.

Además, los espacios $W_{o}^{1,2}(\Omega, a)$ y $W_{o}^{1,2}(\Omega, b)$ definidos como la clausura de $C_{o}^{\infty}(\Omega)$ con respecto a estas normas son espacios de Hilbert.

Demostración. Primero, probaremos que $\|\cdot\|_{a}$ está bien definida. Si $u \in C_{o}^{\infty}(\Omega)$ entonces $\frac{\partial u}{\partial x_{i}} \in C_{o}^{\infty}(\Omega)$, además $\operatorname{sopp}\left(\frac{\partial u}{\partial x_{i}}\right) \subset \operatorname{sopp}(u)$. Por tanto, $K=\operatorname{sopp}\left(|\nabla u|^{2}\right) \subset \operatorname{sopp}(u)$ implica

$$
\|u\|_{a}^{2}=\int_{\Omega} a(x)|\nabla u|^{2} d x \leq \operatorname{máx}_{x \in K}|\nabla u|^{2} \int_{K} a(x) d x<\infty .
$$

Así, $\|\cdot\|_{a}$ está bien definida. Análogamente $\|\cdot\|_{b}$ está bien definida.

Probemos que $\|\cdot\|_{a}$ es una norma:

(i) $\|u\|_{a}^{2}=\int_{\Omega} a(x)|\nabla u|^{2} d x \geq 0$ ya que $a(x)>0 \mathrm{y}|\nabla u|^{2} \geq 0$.

(ii) $\|u\|_{a}^{2}=0$ implica $u=0$ en c. t. p. de $\Omega$.

En efecto, para $p=2$ la desigualdad de Friedrichs con peso, es dada por:

$$
\|u\|_{L^{2}(\Omega)}^{2}=\int_{\Omega}|u(x)|^{2} d x \leq c_{4} \int_{\Omega} a(x)|\nabla u(x)|^{2} d x=c_{4}\|u\|_{a}^{2},
$$

ver la ecuación (18) de la Observación 2.4 en la Sección anterior. Si $\|u\|_{a}=0$, la desigualdad anterior implica $\|u\|_{L^{2}(\Omega)}^{2}=0$, luego $u=0$ en c.t.p. de $\Omega$.

(iii) $\|\alpha u\|_{a}^{2}=\int_{\Omega} a(x)|\alpha \nabla u|^{2}=\alpha^{2} \int_{\Omega} a(x)|\nabla u|^{2} d x=\alpha^{2}\|u\|_{a}^{2}$.

(iv) $\|u+v\|_{a}=\left[\int_{\Omega} a(x)|\nabla(u+v)|^{2} d x\right]^{1 / 2} \leq\left[\int_{\Omega} a|\nabla u|^{2}+\int_{\Omega} a|\nabla v|^{2}+2 \int_{\Omega} a|\nabla u||\nabla v|\right]^{1 / 2}$ luego por la desigualdad de Hölder

$$
\int_{\Omega} a|\nabla u||\nabla v| d x=\int_{\Omega} a^{1 / 2}|\nabla u| a^{1 / 2}|\nabla v| d x \leq\|u\|_{a}\|v\|_{a}, \text { esto es, }
$$




$$
\|u+v\|_{a} \leq\left[\|u\|_{a}^{2}+\|v\|_{a}^{2}+2\|u\|_{a}\|v\|_{a}\right]^{1 / 2}=\|u\|_{a}+\|v\|_{a} .
$$

Así $\|\cdot\|_{a}$ es una norma. De la misma forma se prueba que $\|\cdot\|_{b}$ es una norma. Veamos ahora para $u \in W_{o}^{1,2}(\Omega, a)$, esto es, si $u \in{\overline{C_{o}^{\infty}(\Omega)}}^{\|\cdot\|_{a}}$ entonces $\exists\left(\varphi_{n}\right) \subset C_{o}^{\infty}(\Omega)$ tal que $\varphi_{n} \longrightarrow u$ luego definimos

$$
\|u\|_{a}=\lim _{n \longrightarrow \infty}\left\|\varphi_{n}\right\|_{a} .
$$

La buena definición se verifica debido a la equivalencia de las normas $\|\cdot\|_{a} \mathrm{y}\|\cdot\|_{W^{1,2}(\Omega, a)}$ en $C_{o}^{\infty}(\Omega) \subset W_{o}^{1,2}(\Omega, a) \subset W^{1,2}(\Omega, a)$, esto es,

$$
c_{1}\left\|\varphi_{n}\right\|_{W^{1,2}(\Omega, a)} \leq\left\|\varphi_{n}\right\|_{a} \leq c_{2}\left\|\varphi_{n}\right\|_{W^{1,2}(\Omega, a)} .
$$

De esto y los resultados anteriores, se verifica las condiciones de norma $\|\cdot\|_{a}$.

Se demuestra de manera similar que estas normas $\|\cdot\|_{a},\|\cdot\|_{b}$ provienen de un producto interno $(\cdot, \cdot)_{a},(\cdot, \cdot)_{b}$ respectivamente, definidos por

$$
\left(f_{1}, g_{1}\right)_{a}=\int_{\Omega} a(x) \nabla f_{1} \cdot \nabla g_{1} d x, \forall f_{1}, g_{1} \in C_{o}^{\infty}
$$

$\mathrm{y}$

$$
\left(f_{2}, g_{2}\right)_{b}=\int_{\Omega} b(x) \nabla f_{2} \cdot \nabla g_{2} d x, \forall f_{2}, g_{2} \in C_{o}^{\infty} .
$$

Además, $W_{o}^{1,2}(\Omega, a)$ y $W_{o}^{1,2}(\Omega, b)$ son espacios de Hilbert. Para probar esto recordemos que por definición

$$
W_{o}^{1,2}(\Omega, a):=\overline{C_{o}^{\infty}(\Omega)} \subset W_{o}^{1,2}(\Omega, a),
$$

luego como $W^{1,2}(\Omega, a)$ es un espacio completo y $W_{o}^{1,2}(\Omega, a)$ es un subespacio cerrado, entonces $W_{o}^{1,2}(\Omega, a)$ es completo. También se tiene que esta norma $\|\cdot\|_{a}$ proviene de un producto interno en este espacio, por lo tanto $W_{o}^{1,2}(\Omega, a)$ es un espacio de Hilbert. Procediendo en forma análoga obtenemos el espacio de Hilbert $W_{o}^{1,2}(\Omega, b)$.

Definimos el espacio de Hilbert $W:=W_{o}^{1,2}(\Omega, a) \times W_{o}^{1,2}(\Omega, b)$, bajo la norma:

$$
\|(u, v)\|_{W}=\|u\|_{a}+\|v\|_{b}
$$

y con producto escalar:

$$
\langle f, g\rangle=\int_{\Omega}\left(a(x) \nabla f_{1} \nabla g_{1}+b(x) \nabla f_{2} \nabla g_{2}\right) \forall f=\left(f_{1}, f_{2}\right), g=\left(g_{1}, g_{2}\right) \in W
$$

Entonces $W$ es un espacio uniformemente convexo ya que $W$ es un espacio de Hilbert.

Para probar que $W$ es un espacio Hilbert bastará con recordar que el producto cartesiano de los espacios de Hilbert $W_{o}^{1,2}(\Omega, a)$ y $W_{o}^{1,2}(\Omega, b)$ es un espacio de Hilbert.

Proposición 3.2 La inmersión continua

$$
W \hookrightarrow\left(W^{1,2_{s}}(\Omega)\right)^{2} \text { vale para } 2_{s}=\frac{2 s}{s+1}
$$

Demostración. La demostración es análoga a la demostración realizada en la Afirmación 1

Proposición 3.3 Se tiene la inmersión de Sobolev

$$
W \hookrightarrow\left(L^{2_{s}^{*}}(\Omega)\right)^{2} .
$$


Demostración. La prueba sigue del diagrama

$$
W \hookrightarrow\left(W^{1,2_{s}}(\Omega)\right)^{2} \hookrightarrow\left(L^{2_{s}^{*}}(\Omega)\right)^{2}
$$

y de la inmersión continua $W^{1, p}(\Omega) \hookrightarrow L^{q}(\Omega)$ donde $\frac{1}{q}=\frac{1}{p}-\frac{1}{N}, p<N$.

Proposición 3.4 Tenemos la inmersión compacta

$$
W \hookrightarrow L^{r}(\Omega) \times L^{t}(\Omega) \text { donde } 1 \leq r, t<2_{s}^{*} .
$$

Demostración. La prueba sigue del diagrama

$$
W \hookrightarrow\left(W^{1,2_{s}}(\Omega)\right)^{2} \hookrightarrow\left(L^{r}(\Omega) \times L^{t}(\Omega)\right) \text { para } 1 \leq r, t<2_{s}^{*}
$$

y de la inmersión compacta

$$
p<N, W^{1, p}(\Omega) \hookrightarrow L^{q}(\Omega) \forall 1 \leq q<p^{*} \text { donde } \frac{1}{p^{*}}=\frac{1}{p}-\frac{1}{N}
$$

Observación 3.1 Del sistema elíptico semilineal (1), consideremos

$$
-\operatorname{div}(a \nabla u)=\lambda F_{u}(x, u, v) \text { en } \Omega \text { con } u=0 \text { en } \partial \Omega .
$$

De las propiedades de la divergencia se consigue

$$
-\operatorname{div}(a \nabla u)=-\nabla \cdot(a \nabla u)
$$

multiplicando por $\varphi$ a la igualdad (21), integrando sobre $\Omega$ y aplicando el teorema de Green al término de la derecha tenemos:

$$
-\int \operatorname{div}(a \nabla u) \varphi d x=-\int[\nabla \cdot(a \nabla u)] \varphi d x=\int[a \nabla u] \nabla \varphi d x .
$$

De manera similar obtenemos que

$$
-\int_{\Omega} \operatorname{div}(b \nabla v) \psi d x=\int b \nabla v \nabla \psi d x
$$

Luego

$$
\begin{gathered}
\int_{\Omega} a \nabla u \nabla \varphi d x=\lambda \int_{\Omega} F_{u}(x, u, v) \varphi d x \mathrm{y} \\
\int_{\Omega} b \nabla v \nabla \psi d x=\lambda \int_{\Omega} F_{v}(x, u, v) \psi d x
\end{gathered}
$$

entonces sumando los resultados anteriores

$$
\int_{\Omega}(a \nabla u \nabla \varphi+b \nabla v \nabla \psi) d x=\lambda \int_{\Omega}\left[F_{u}(x, u, v) \varphi+F_{v}(x, u, v) \psi\right] d x \forall(\varphi, \psi) \in W
$$

Definición 3.1 Decimos que $(u, v) \in W$ es una solución débil del sistema (1) si y solo si satisface (22), esto es:

$$
\int_{\Omega}(a(x) \nabla u \nabla \varphi+b(x) \nabla v \nabla \psi) d x=\lambda \int_{\Omega}\left[F_{u}(x, u, v) \varphi+F_{v}(x, u, v) \psi\right] d x, \forall(\varphi, \psi) \in W .
$$

Así, las soluciones débiles del problema (1) son las soluciones de la ecuación integral (22). Definimos el funcional correspondiente asociado al problema(1) como:

$$
\Im_{\lambda}(u, v)=\frac{1}{2} \int_{\Omega}\left(a(x)|\nabla u|^{2}+b(x)|\nabla v|^{2}\right) d x-\lambda \int_{\Omega} F(x, u, v)
$$


El funcional $\Im_{\lambda}(u, v)$ está bien definido, es de clase $C^{1}(W)$ y por tanto las soluciones débiles de (1) son exactamente los puntos críticos del funcional $\Im_{\lambda}(u, v)$ que tiene como derivada débil:

$$
\left\langle\Im_{\lambda}^{\prime}(u, v),(\varphi, \psi)\right\rangle=\int_{\Omega}(a \nabla u \nabla \varphi+b \nabla v \nabla \psi) d x-\lambda \int_{\Omega}\left[F_{u}(x, u, v) \varphi-F_{v}(x, u, v) \psi\right] d x
$$

Teorema 3.1 (Primer teorema de existencia) Suponga que la condición $\left(F_{1}\right)$ es satisfecha. Entonces existe una constante $\underline{\lambda}>0$ tal que para todo $0<\lambda<\underline{\lambda}$, el sistema (1) tiene una solución débil.

Este teorema es demostrado mediante el Principio del Mínimo y los lemas 3.2 y 3.3:

Lema 3.2 El funcional $\Im_{\lambda}$ dado por (23) es débilmente semicontinuo inferiormente en $W$.

Demostración. Ver [1] lema 2.1.

Lema 3.3 El funcional $\Im_{\lambda}$ dado por (23) es coerciva y acotada inferiormente en $W$.

Demostración. Ver [1] lema 2.2

Los lemas 3.2 y 3.3 verifican las hipótesis del Principio del Mínimo, por tanto el funcional $\Im_{\lambda}$ alcanza su mínimo en $W$, además como el funcional (23) es diferenciable podemos concluir que el sistema (1) admite al menos una solución débil.

Enunciamos y probamos el segundo teorema de existencia de soluciones.

Teorema 3.4 En adición al Primer teorema de existencia, suponga que las condiciones $\left(F_{1}\right)-$ $\left(F_{4}\right)$ son satisfechas. Entonces el problema (1) tiene una solución débil no trivial.

Este teorema es demostrado mediante el Teorema del Paso de la montaña y los lemas 3.5 y 3.6:

Lema 3.5 El funcional $\Im_{\lambda}$ dado por (23) satisface la condición de Palais smale en $W$.

Demostración. Ver [1] lema 3.1.

El Lema 3.5 nos dice que el funcional $\Im_{\lambda}$ satisface la condición de Palais-Smale (Condición de compacidad).

Lema 3.6 Bajo las hipotesis $(F 1)$ - (F4) el funcional $\Im_{\lambda}$ dado por (23) satisface:

(i) Existen $\rho, \sigma>0$ tal que $\|(u, v)\|_{H}=\rho$ implica $\Im(u, v) \geq \sigma>0$.

(ii) Existe $\left(u_{o}, v_{o}\right) \in W$ tal que $\left\|\left(u_{o}, v_{o}\right)\right\|_{H}>\rho y \Im\left(u_{o}, v_{o}\right) \leq 0$.

Demostración. Ver [1] lema 3.2.

El lema 3.6 verifica que el funcional $\Im_{\lambda}$ tiene la geometría del Teorema del paso de la montaña. Es así que el funcional $\Im_{\lambda}$ satisface las hipótesis del Teorema del paso de la montaña, por tanto tiene un punto crítico no cero y el punto crítico no cero de $\Im_{\lambda}$ es precisamente la solución débil no trivial del problema (1).

\section{Un ejemplo de aplicación}

Consideremos el siguiente ejemplo de un sistema elíptico semilineal potencial :

$$
\text { Sea } N=3,, s \in\left(\frac{3}{2} ; \infty\right) \text { y } 1,2<2_{s}<2,2<2_{s}^{*}<6 .
$$


Con una elección particular de los pesos (funciones medibles no negativos sobre $\Omega$ )

$$
\begin{gathered}
a(x, y, z)=1+x^{2} \\
b(x, y, z)=1+y^{2}
\end{gathered}
$$

y el funcional

$$
F((x, y, z), u, v)=u^{2,2} \cdot v^{2,5}
$$

Obtenemos el siguiente sistema:

$$
\left\{\begin{array}{rr}
-\operatorname{div}\left(\left|1+x^{2}\right| \nabla u(x, y, z)\right)=2,2 \cdot u^{1,2} \cdot v^{2,5}, & (x, y, z) \text { en } \Omega \\
-\operatorname{div}\left(\left|1+y^{2}\right| \nabla v(x, y, z)\right)=2,5 \cdot u^{2,2} \cdot v^{1,5}, & (x, y, z) \text { en } \Omega \\
\mathrm{u}=\mathrm{v}=0 & \text { sobre } \partial \Omega
\end{array}\right.
$$

donde $\Omega$ es un dominio acotado en $\mathbb{R}^{3}$, de frontera bien regular,

$$
\left(2,2 u^{1,2} \cdot v^{2,5} ; 2,5 u^{2,2} \cdot v^{1,5}\right)=\left(F_{u}((x, y, z), u, v) ; F_{v}((x, y, z), u, v)\right)=\nabla F((x, y, z), u, v)
$$

representa el gradiente de $F((x, y, z), u, v)=u^{2,2} \cdot v^{2,5}$ en las variables $(u, v) \in \mathbb{R}^{2}$.

Se probará la existencia de al menos una solución débil para el sistema, bajo adecuadas hipótesis sobre el dato:

$$
\begin{array}{ccc}
F: \begin{array}{c}
\Omega \times \mathbb{R} \times \mathbb{R} \\
((x, y, z), u, v)
\end{array} & \mapsto & \mathbb{R} \\
& \mapsto((x, y, z), u, v)=u^{2,2} \cdot v^{2,5}
\end{array}
$$

es un $C^{1}$ - funcional que satisface :

(F1) Existen constantes positivas $c_{1}=2,2, c_{2}=2,5$ tal que :

$$
\begin{aligned}
& \left|F_{u}((x, y, z), u, v)\right| \leq c_{1}|u|^{1,2}|v|^{2,5} \\
& \left|F_{v}((x, y, z), u, v)\right| \leq c_{2}|u|^{2,2}|v|^{1,5}
\end{aligned}
$$

$\forall(u, v) \in \mathbb{R}^{2}$, en casi todo punto de $\Omega$ y algún $\gamma=1,2>1, \delta=1,5>1$ tales que

$\frac{1,2+1}{p}+\frac{1,5+1}{q}=1$ y $1,2+1<p<2_{s}^{*} \leq 6,1,5+1<q<2_{s}^{*} \leq 6$; podemos tomar $p=\frac{2,2 \cdot q}{q-2,5}$ y hacer $q \stackrel{q}{=} 4$ y $p=5,86$.

(F2) Existen constantes positivas $c=\frac{1}{1,8}, \alpha=2,2 \cdot 1,8=3,96$ y $\beta=2,25 \cdot 2,25=5,0625$, con $2<\alpha, \beta<2_{s}^{*}$ tal que

$$
|F((x, y, z), u, v)| \leq c\left(1+|u|^{\alpha}+|v|^{\beta}\right)
$$

para todo $(x, y, z) \in \bar{\Omega}$ y $u, v$ en $\mathbb{R}$.

( Para este resultado se utilizó la desigualdad de Young: $u^{2,2} v^{2,5} \leq \frac{1}{A} u^{2,2 A}+\frac{1}{B} v^{2,5 B}$ con $\frac{1}{A}+\frac{1}{B}=1$ para $A=1,8$ y $\left.B=2,25\right)$.

(F3) Existen $R=1 / 2>0, \theta=0,3280$ y $\theta^{\prime}=0,2127$ con $1 / 6 \leq \frac{1}{2_{s}^{*}}<\theta, \theta^{\prime}<\frac{1}{2}$ tal que

$$
0<F(x, u, v) \leq \theta u F_{u}(x, u, v)+\theta^{\prime} v F_{v}(x, u, v)
$$

para todo $x \in \bar{\Omega}$ y $|u|,|v| \geq R$.

(Para este resultado se utilizó el Teorema del valor medio: $u^{2,2} v^{2,5}=u F_{u}(x, r u, r v)+$ $v F(x, r u, r v)=2,2 r^{3,7} F(x, u, v)+2,5 r^{3,7} F(x, u, v)$

$\left.\leq 0,3280 u \cdot F_{u}(x, u, v)+0,2127 v \cdot F_{v}(x, u, v)\right)$. 
(F4) Existe $c=1 / 1,8, \bar{\alpha}=3,96>2, \bar{\beta}=5,0625>2$ y $\epsilon=1 / 2>0$ tal que

$$
|F(x, u, v)| \leq c\left(|u|^{\bar{\alpha}}+|v|^{\bar{\beta}}\right)
$$

para todo $x \in \bar{\Omega} \mathrm{y}|u| \leq \epsilon,|v| \leq \epsilon$.

El sistema elíptico semilineal, mostrado satisface las condiciones enunciadas y está dentro de la clase de sistemas elípticos semilineales tratados, por tanto concluimos que en aplicación a los teoremas de existencia de soluciones, se comprueba que existe al menos una solución débil no trivial al problema (25).

\section{Conclusión}

Para esta clase de sistemas elípticos semilineales caracterizado por la no linealidad $F(x, u, v)$ es posible probar la existencia de soluciones débiles no triviales mediante el Teorema del paso de la montaña y el Principio del Mínimo, esto gracias a las condiciones (F1) - (F4).

Primero, podemos concluir mediante el Principio del Mínimo que existe un mínimo $\left(u_{1}, v_{1}\right) \in W$ tal que

$$
\Im_{\lambda}\left(u_{1}, v_{1}\right)=\inf _{(u, v) \in W} \Im_{\lambda}(u, v) .
$$

Como el funcional $\Im_{\lambda}$ es diferenciable en $W$, el punto $\left(u_{1}, v_{1}\right) \in W$ de mínimo es un punto crítico de $\Im_{\lambda}$, esto es, $\Im_{\lambda}^{\prime}\left(u_{1}, v_{1}\right)=0$ y así $\left(u_{1}, v_{1}\right)$ es una solución débil del problema. Aún mas, debido al lema 3.6 item(ii) existe $\left(u_{0}, v_{0}\right) \in W$ tal que $\left\|\left(u_{0}, v_{0}\right)\right\|_{W}>\rho$ y $\Im_{\lambda}\left(u_{0}, v_{0}\right)<0$ para $0<\lambda<\underline{\lambda}$, podemos concluir que $\left(u_{1}, v_{1}\right)$ es una solución débil no trivial del problema.

En segundo lugar, el Teorema del paso de la montaña concluye para el nivel minimax $c$

$$
0<\sigma \leq c=\inf _{g \in \Gamma} \operatorname{máx}_{(u, v) \in g([0,1])} \Im_{\lambda}(u, v)
$$

donde $\Gamma=\left\{g \in C([0,1], W) / g(0)=(0,0), g(1)=\left(u_{0}, v_{0}\right)\right\}$, así para el valor crítico $c$ existe un punto crítico $\left(u_{2}, v_{2}\right) \in W$ segunda solución no trivial.

\section{Referencias bibliográficas}

[1] Afrouzi, G., Mirzapour, A. and Zographopoulos, N. (2012). Existence results for a class of semilinear elliptic systems. Theoretical Mathematics \& Applications, 2, (2), 77 - 86.

[2] Alves, C. (2007). Local mountain pass for a class of elliptic system. Journal of Mathematical Analysis and Applications, 335 (1) 135-150.

[3] Boccardo, L., and De Figueiredo, D. (2000) Some remarks on a system of quasilinear elliptic equations. Nonlinear Differential Equations and Applications, 9 (7), 309-323.

[4] Costa, D. (1994). On a class of elliptic system in $\mathbb{R}^{N}$. Electronic Journal of Differential Equations, 7, 1-14.

[5] Caldiroli, P. y Musina, R. (2000). On a variational degenerate elliptic problem. Nonlinear Differential Equations and Applications, 7 (2), 187-199.

[6] Chung, N. and Toan, H.(2009) On a class of degenerate and singular elliptic systems in bounded domain.Journal of Mathematical Analysis and Applications, 360, (2), 422-431. 
[7] Dautray, R. y Lions, J. (1985). Mathematical Analysis and Numerical Methods for Science and Technology I: Physical Origins and Classical Methods. Berlin: Springer-Verlag.

[8] Drabek, P., Kufner, A. and Nicolosi, F. (1997). Quasilinear elliptic equations with degenerate and singularities. Berlin: Walter de Gruyter.

[9] Djellit, A. and Tas, S. (2003). Existence of solutions for a class of elliptic systems in $\mathbb{R}^{N}$ involving the p-Laplacian. Electronic Journal of Differential Equations, 56, 1-8.

[10] Kivshar, Y., \& Agrawal, G. (2003). Optical Solitons: From Fibers to Photonic crystals. New York: Academic Press.

[11] Zeidler, E. (1985). Nonlinear Functional Analysis and its applications. Vol. 1 Fixed-Point Theorems. Berlin: Springer-Verlag.

[12] Zographopoulos, N. (2004). p-Laplacian systems on resonance. Applicable analysis, 83 (5), 509-519.

[13] Tineo, M. (2017). Existencia de Soluciones para una Clase de Sistemas Elípticos Semilineales. Tesis de Maestria en Matemática Pura. Universidad Nacional Mayor de San Marcos, Lima, Perú.

[14] Zhang, G. y Wang, Y. (2007). Some existence results for a class of degenerate semilinear elliptic system. Journal of Mathematical Analysis and Applications, 333 (2), 904-918. 